\title{
纤维素二聚体模型物热裂解的热力学性质研究
}

\author{
蒋原野 ${ }^{a}$ 于海珠 $^{b} \quad$ 傅尧*,a \\ ( ${ }^{a}$ 中国科学技术大学化学系 合肥 230026) \\ ( ${ }^{b}$ 北京科技大学高分子科学与工程系 北京 100083 )
}

\begin{abstract}
摘要 纤维素是生产生物质燃料, 精细化工品的重要原料. 热解是由纤维素解聚的重要手段之一. 了解纤维素热解的 反应机理对发展高效的生物质转化技术具有重要意义. 我们利用密度泛函理论方法, 以纤维素二聚体为模型, 详细研 究了其发生键均裂过程的热力学能量变化. 发现糖苗键和侧链 $\mathrm{C}-\mathrm{C}$ 键的均裂相对于 $\mathrm{C}-\mathrm{OH}$ 键和 $\mathrm{O}-\mathrm{H}$ 键均裂在热力 学上更优. 此外, 我们发现不同物种的热力学稳定性与其在纤维素快速热解实验产物中的比例无关. 最后我们发现温 度对反应能否自发发生具有重要的影响, 为通过调节温度来改变不同类型反应的选择性提供了一条思路.
\end{abstract}

关键词 纤维素二聚体; 热裂解; 密度泛函理论方法

\section{Theoretical Study on Thermodynamic Properties of Pyrolysis of Cellulose Dimer Model Compound}

\author{
Jiang, Yuanye ${ }^{a} \quad \mathrm{Yu}$, Haizhu $^{b} \quad \mathrm{Fu}, \mathrm{Yao}^{*, a}$ \\ ( ${ }^{a}$ Department of Chemistry, University of Science and Technology of China, Hefei 230026) \\ ( ${ }^{b}$ Department of Polymer Science and Engineering, University of Science and Technology Beijing, Beijing 100083)
}

\begin{abstract}
Cellulose is an important material for production of biofuel and refined chemicals. Pyrolysis is one of the most promising approaches for cellulose de-polymerization. Understanding the mechanism of cellulose pyrolysis is essential for development of efficient biomass conversion technologies. In this study, the thermodynamic energy change of cellulose pyrolysis through homolytic bond cleavage was studied with the aid of density functional theory method by using cellulose dimer as a model compound. The free energy changes of various homolytic bond dissociation of cellulose dimer were studied by the method of M06-2x at the temperature of $800{ }^{\circ} \mathrm{C}$. To compare with experiment results of cellulose pyrolysis reported recently by Huber et al., the free energy changes of reaction pathways studied by Auerbach group via Car-Parrinello molecular dynamics calculations were also studied. Calculated results show that the free energy changes of homolytic dissociation of glucosidic bond varies in the range of $45 \sim 51 \mathrm{kcal} / \mathrm{mol}$. The free energy changes of homolytic bond dissociation of C-OH bond vary in the range of $62 \sim 70 \mathrm{kcal} / \mathrm{mol}$. The free energy changes of homolytic bond dissociation of $\mathrm{O}-\mathrm{H}$ bond vary in the range of $82 \sim 95 \mathrm{kcal} / \mathrm{mol}$. The free energy changes of homolytic bond dissociation of $\mathrm{C}-\mathrm{C}$ bond vary around 46 $\mathrm{kcal} / \mathrm{mol}$. Furthermore, thermodynamic stabilities of products were found to be irrelevant to the ratios of them in Huber et al.'s fast pyrolysis experiments no matter at a high temperature $(800 \mathrm{~K})$ or a mild temperature $(298 \mathrm{~K})$. Finally, we found that temperature have a significant influence on whether a reaction can occur spontaneously or not. At relatively high temperature, the reactions having significant increase of entropy are favored, e.g. dehydration. The free energy of reactions with small entropy changes are less sensitive to the change of temperature. This foundation provides an inspiration for controlling chemical selectivity of different types of reactions by temperature regulation.

Keywords cellulose dimer; pyrolysis; density functional theory
\end{abstract}

\section{1 引言}

纤维素是自然界中含量最丰富的可再生生物质. 除 了被大量用以生产纸张外 ${ }^{[1]}$, 纤维素还被用以生产生物 质燃料及精细化工品 ${ }^{[2]}$. 在该研究方向上, 热解是降解 纤维素, 产生可燃烧或可化学转化小分子的一种重要手
段 ${ }^{[3]}$. 了解纤维素的热解过程对发展高效的生物质转化 技术具有重要意义. 实验上主要采用热分析手段来确定 裂解性质, 建立裂解动力学模型加以分析 ${ }^{[4]}$. 然而纤维 素热解是一个复杂过程, 涉及多相反应 ${ }^{[5]}$ 、复杂化学机 理 ${ }^{[6]}$ 和不稳定中间体 ${ }^{[7]}$, 给实验分析带来不便. 理论计

\footnotetext{
*E-mail: fuyao@ustc.edu.cn; Tel.: 0551-63607476; Fax: 0551-63606689

Received July 22, 2013; published October 30, 2013.
}

Supporting information for this article is available free of charge via the Internet at http://sioc-journal.cn.

Project supported by the National Natural Science Foundation of China (Nos. 21172209, 21272223, 21202006), the Specialized Research Fund for the Doctoral Program of Higher Education (No. 20123402110051), the Fundamental Research Funds for the Central Universities (Nso. WK2060190008, FRF-TP-13-023A) and the Chinese Academy of Sciences (No. KJCX2-EW-J02).

项目受国家自然科学基金(Nos. 21172209,21272223, 21202006)、高等学校博士学科点专项科研基金资助课题(No. 20123402110051)、中央高校基本科 研业务费专项资金(No. WK2060190008, FRF-TP-13-023A)和中国科学院基金(No. KJCX2-EW-J02)资助. 
算由于可以提供直观的分子水平上的研究, 近年来受到 了研究者们的重视, 并被应用到化合物热解机理的研究 中 $^{[8]}$.

最近 Zhang 等 ${ }^{[9]}$ 利用密度泛函理论(DFT)方法对纤 维素热解产生左旋葡聚糖(LGA)和甲醛的机理进行了研 究. Auerbach 等 ${ }^{[10]}$ 利用分子动力学方法模拟了纤维素分 别在 327 和 $600{ }^{\circ} \mathrm{C}$ 条件下的热降解过程, 并估算了产生 甲酸和 5-羊圣甲基糠醛(HMF)、左旋葡聚糖(LGA)前体的 过程的能垒. 前人在确定纤维素反应历程时采取直接计 算可能的反应路径能垒加以比较的办法. 该方法虽然更 为准确, 但相较于常见热力学性质的计算更加耗时. 因 而对于复杂的化学过程, 通常只采取该方法研究其中特 定反应过程加以比较. 此外, 该方法因为需要确定反应 中的过渡态结构, 对使用者的专业技术要求更高, 而不 能被更多的化学工作者所利用.

纤维素热解通常是在高温条件下进行, 而常见有 机反应往往在 $200{ }^{\circ} \mathrm{C}$ 以下进行. 所以常见的有机反应 原则上在纤维素的热解温度下都可以发生. 这种情况下, 各种组分之间的转化在动力学上是允许的, 最终各组分 的比例很大程度上取决于各组分的热力学稳定性和反 应进行程度. 事实上已有研究指出热力学性质是可能与 反应活性或产物比例相关的 ${ }^{[11]}$. 本工作中, 我们以纤维 素二聚体为模型化合物, 研究其热解过程可能涉及的中 间体或产物的热力学稳定性. 在此基础上, 我们对比前 人的热解实验结果, 检验热力学稳定性是否能用以预测 产物成分比例.

\section{2 计算模型与方法}

参考 Huber 等 ${ }^{[10]}$ 的工作, 我们选取纤维素二聚体 $\mathbf{A}$ (图 1)作为纤维素的模型物, 研究其热解过程的热力学 性质. 所有计算利用 Gaussian 09 软件包进行 ${ }^{[12]}$. 结构 优化采用密度泛函方法 M06-2x ${ }^{[13]}$ 在真空气象中进行, 所有原子采用 6-31G(d)基组. 在同等计算水平下进行 热力学分析, 确保优化所得结构没有虚频, 为能量极 小值点, 并获取对吉布斯自由能的热力学矫正能 $\Delta G_{\mathrm{cor}}$. 对优化所得结构采取更高水平的计算方法 M06-2x/ $6-311++\mathrm{G}(2 \mathrm{df}, 2 \mathrm{p})$ 计算其单点能 $E^{[14]}$. 下文所讨论的 所有物种的自由能 $G$ 等于 $E$ 加上 $\Delta G_{\text {cor }}$, 反应的自由能 变 $\Delta G$ 等于产物的自由能减去反应物的自由能. 在获 知化合物的热力学稳定性之后, 我们将其与 Huber 的 纤维素快速热解实验结果进行对比. 实验中反应温度 由从 $323 \mathrm{~K}$ 开始以每分钟升高 $150 \mathrm{~K}$ 的速率加热至 $1073 \mathrm{~K}$, 然后在 $1073 \mathrm{~K}$ 的条件下再维持 $6 \mathrm{~min}$. 考虑到 整个反应可能在升温过程中也会发生, 我们选取了中 间偏高的温度 $(800 \mathrm{~K})$ 和 $101 \mathrm{kPa}$ 作为计算的默认条件.

\section{3 结果与讨论}

前人在研究反应机理时, 考察了糖苷键均裂过程,
而其它一些类型的键均裂过程没有被研究过. 本工作中 我们以纤维素二聚体为模型, 首先系统研究其发生 $\mathrm{C}$ $\mathrm{O}, \mathrm{O}-\mathrm{H}$ 和 $\mathrm{C}-\mathrm{C}$ 键均裂过程的反应能变以及后续可能 产生的中间体的热力学稳定性, 以完善对纤维素二聚体 热解机理的认识.

\section{$3.1 \mathrm{C}$ - $\mathrm{O}$ 键断裂}

我们首先考虑纤维素二聚体侧链上的 $\mathrm{C}-\mathrm{O}$ 键发生 均裂, 形成碳自由基和羟基自由基的情况(图 2). 二聚体 $\mathbf{A}$ 首先经历上述过程, 可以分别形成 $\mathbf{B} n(n=1 \sim 8)$ 这 8 种碳自由基中间体，对应过程的自由能分布在 $62 \sim 70$ $\mathrm{kcal} / \mathrm{mol}$. 有趣的是, 在这 8 种均裂方式中, 产生一级碳 自由基 B7 的均裂方式反而比其它产生二级碳自由基的 均裂方式的能量需求更低. 这可能是因为形成 B7 断裂 的并非环上的 $\mathrm{C}-\mathrm{O}$ 键, 对分子核心的六元环骨架结构 的影响相对较少.

碳自由基中间体 B1 可以通过脱去氢原子给羟基自 由基，形成水和酮 B1a 或烯烃 B1b, 对应过程的自由能变 分别为 -97.6 和 $-90.1 \mathrm{kcal} / \mathrm{mol}$, 形成酮 B1a 更加有利. B2 可以经历类似过程可以形成两种酮 B2a 或 B2b. 在 $\mathbf{B} 2 \mathbf{a}$ 和 $\mathbf{B} 2 \mathbf{b}$ 相比, B2a 不存在 C(4)和 C(6) 上羟基的氢键作 用, 而 $\mathbf{B} 2 \mathbf{b}$ 则没有 $\mathrm{C}(2)$ 和 $\mathrm{C}(3)$ 上羟基的氢键作用. 由 $\mathbf{B} 2$ 形成 B2a 和 $\mathbf{B} 2 \mathbf{b}$ 的自由能变化分别为 -93.3 和 -87.7 $\mathrm{kcal} / \mathrm{mol}$, 前者更优. $\mathbf{B 3}$ 可以脱去 $\mathrm{C}(5)$ 上的氢原子形成烯 烃 B3a, 对应的自由能变化为一 $83.1 \mathrm{kcal} / \mathrm{mol}$. 最后我们 考虑了 B4 形成酮 B4a 的过程，该过程自由能降低-91.6 $\mathrm{kcal} / \mathrm{mol}$. 与上述过程类似的, $\mathbf{B} \boldsymbol{n}(n=5 \sim 8)$ 也可以分别 形成酮或烯烃等分子 $\mathbf{B n a}$ 或 $\mathbf{B} \boldsymbol{n b}(n=5 \sim 8)$. 在上面各种 碳自由基向中间产物转化的过程中，形成酮比形成烯烃 普遍在热力学上要普遍占优. 结合均裂形成碳自由基 $\mathbf{B n}$ $(n=1 \sim 8)$ 过程的自由能变化, 计算显示由 $\mathbf{A}$ 形成酮 $\mathbf{B 6 b}$ 最有利, 总反应自由能变化为 $-34.0 \mathrm{kcal} / \mathrm{mol}$ (表 1). 而 由 A 形成烯烃 B1b, B3a 和 B7a 的自由能变比形成 B6b 的自由能变化要高出 $10 \mathrm{kcal} / \mathrm{mol}$ 以上.

表 1 计算所得纤维素二聚体 $\mathbf{A}$ 经历 $\mathrm{C}-\mathrm{OH}$ 键断裂形成中间产物的 自由能变化 $(\mathrm{kcal} / \mathrm{mol})$

Table 1 Calculated free energy change for transformations of cellulose dimer model $\mathbf{A}$ into intermediate products

\begin{tabular}{cc||cc||cc}
\hline Product & $\Delta G$ & Product & $\Delta G$ & Product & $\Delta G$ \\
\hline B1a & -27.8 & B3a & -19.7 & B6a & -29.0 \\
B1b & -20.3 & B4a & -24.3 & B6b & -34.0 \\
B2a & -25.6 & B5a & -24.4 & B7a & -19.6 \\
B2b & -20.0 & B5b & -21.4 & B8a & -20.0 \\
\hline
\end{tabular}

接着我们考察了链接二聚体的糖苷键发生断裂的 情况, 结果如图 3 所示. 二聚体 A 发生糖苷键断裂后可 以分别形成自由基 $\mathrm{B} 9$ 和 $\mathrm{B} 10$ 以及 $\mathrm{B} 11$ 和 $\mathrm{B} 12$, 对应的 自由能变为 +51.4 和 $+44.6 \mathrm{kcal} / \mathrm{mol}$, 后者在热力学上 更优一些. 与图 2 中 $\mathrm{C}-\mathrm{OH}$ 键的断裂相比, 虽然图 3 中 


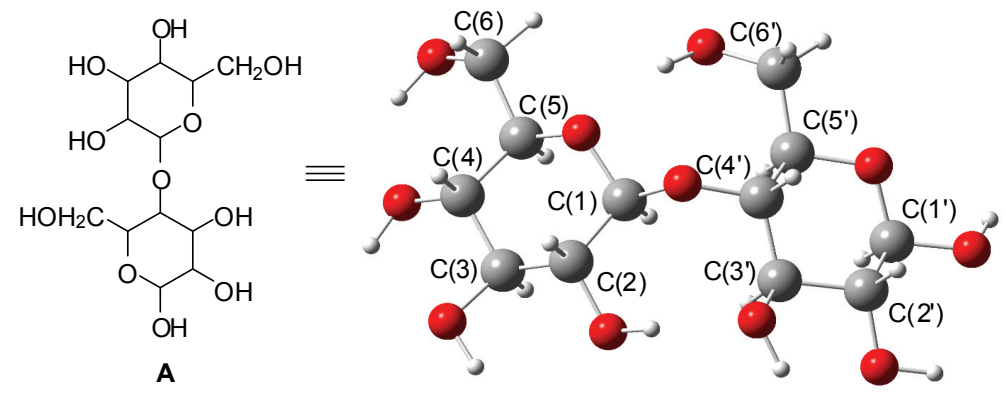

图 1 纤维素二聚体模型化合物

Figure 1 Cellulose dimer model compound

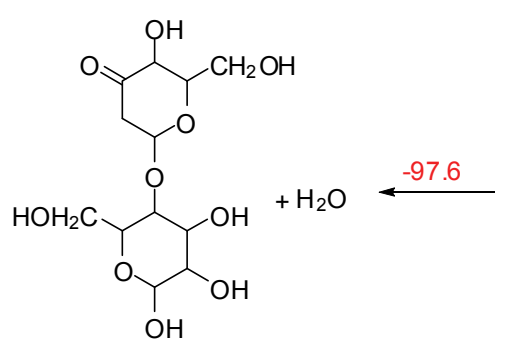

B1a<smiles>OCC1CC(O)C(O)C(OC2C(CO)OC(O)C(O)C2O)O1</smiles>

B4<smiles>C1CCCCC1</smiles><smiles>O=C1CC(CO)OC(OC2C(CO)OC(O)C(O)C2O)C1O</smiles>

B4a<smiles>OCC1OC(OC2C(CO)OC(O)C(O)C2O)CC(O)C1O</smiles>

B1

$+69.8$ $+\cdot \mathrm{OH} \stackrel{-90.1}{\longrightarrow} \mathrm{HOH}_{2} \underbrace{\mathrm{OH}}_{\mathrm{OH}}+\mathrm{H}_{2} \mathrm{O}$

B1b

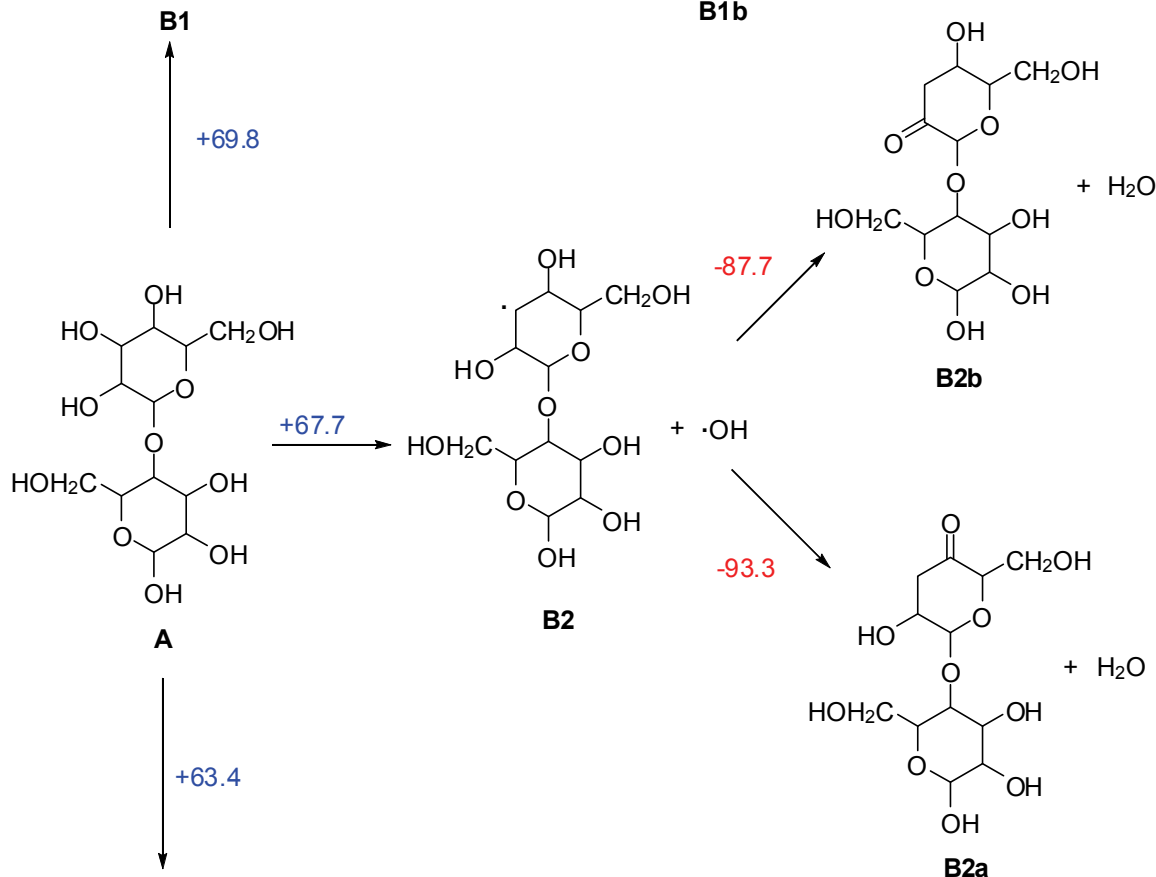<smiles>C=C1OC(OC2C(CO)OC(O)C(O)C2O)C(O)C(O)C1O</smiles>

B3a 


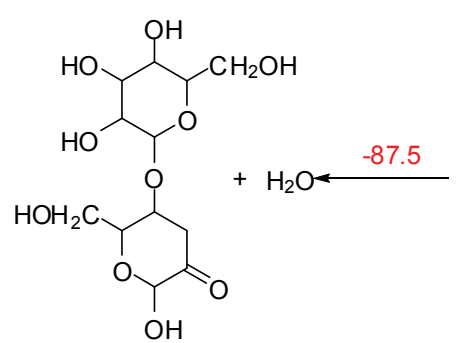

B5a

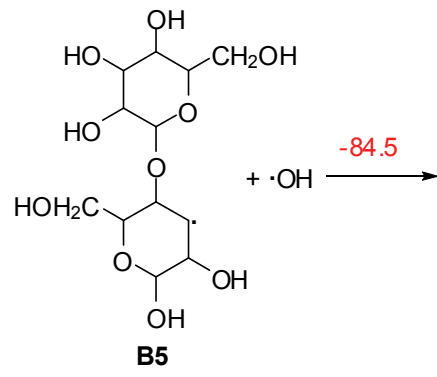

B5<smiles>OCC1OC(O)C(O)C=C1OC1OC(CO)C(O)C(O)C1O</smiles>

B5b<smiles>[R8]C1OC(C)C(OC2OC(CO)C(O)C(O)C2O)C(O)C1O</smiles>

B8<smiles>CC(C)COC(=O)OC1C(OC2OC(CO)C(O)C(O)C2O)OCC(=O)C1O</smiles>

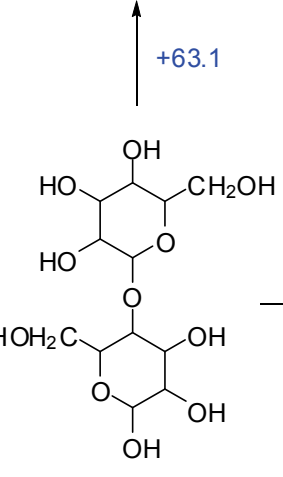

A<smiles>CC1OC(O)C(O)C(O)C1OC1OC(C)C(O[18OH])C(O)C1O</smiles>

B7<smiles>CC(C)(C)[13C](=O)c1ccccc1</smiles>

$+\cdot \mathrm{OH}$<smiles>CC(C)(C)CC1CC(=O)C(OC2OC(CO)C(O)C(O)C2O)C(CO)O1</smiles>

图 2 纤维素二聚体侧链 $\mathrm{C}-\mathrm{O}$ 键断裂过程的自由能 $(\mathrm{kcal} / \mathrm{mol})$ 变化

Figure 2 Free energy $(\mathrm{kcal} / \mathrm{mol})$ change of side chain $\mathrm{C}-\mathrm{O}$ bond cleavage of cellulose dimer model

糖苷键的断裂导致两分子单体之间的氢键作用消失, 但 总体上依然是醚键(糖苷键)的断裂要更加容易. B10 脱 去 C(4)位上的氢原子可以形成酮 B10a，而碳自由基 B9 可以得到一个氢原子形成 $\mathbf{B 9} \mathbf{a}$, 对应自由能变化为 $-73.9 \mathrm{kcal} / \mathrm{mol}$. 另外也可能由 $\mathbf{B 9}$ 脱去 $\mathrm{C}(1)$ 位上的氢原 子, 形成烯醇, 并进一步转化为酮 B9c, 而氧自由基 B10 得到一个氢原子形成醇. 该过程总自由能变化为 -79.4 $\mathrm{kcal} / \mathrm{mol}$. 类似的, B11 和 B12 可以通过氢的转移分别形 成酮 B11a 和醇 B12a 或者酮 B12c 和醇 B11b. 它们对应 的自由能变化分别为 -86.6 和 $-75.7 \mathrm{kcal} / \mathrm{mol}$. 在上述 四种过程中, 由 $\mathbf{A}$ 形成 B11a 和 B12a 的过程的自由能变 最低, 为 $-42.0 \mathrm{kcal} / \mathrm{mol}$. 同时, 对比前面 $\mathrm{C}-\mathrm{OH}$ 键断 裂的计算结果可知, 该过程对于第一步 $\mathrm{C}-\mathrm{O}$ 键均裂和
初级中间产物热力学稳定性上也都是最优势的.

\section{$3.2 \mathrm{O}-\mathrm{H}$ 键断裂}

接着我们考察由二聚体 $\mathbf{A}$ 经历 $\mathrm{O}-\mathrm{H}$ 键均裂形成氧 自由基和氢原子，并进一步转化形成醛或者酮的过程， 如图 4 所示. $\mathrm{A}$ 通过 $\mathrm{O}-\mathrm{H}$ 键均裂可以分别形成 8 种氧 自由基 Bna $(n=13 \sim 20)$, 对应的自由能变化分布在 78 $\sim 86 \mathrm{kcal} / \mathrm{mol}$. 形成的氧自由基可以脱去自由基氧 $\alpha$ 碳上的氢原子形成醛或者酮，同时前一步形成氢自由基 得到该氢原子可以形成氢气. 该过程自由能降低 82 $95 \mathrm{kcal} / \mathrm{mol}$. 综合这两步反应的自由能变, 由 $\mathbf{A}$ 通过 $\mathrm{O}-\mathrm{H}$ 键均裂的途径形成酮或醛的总自由能仅降低 $1 \sim 6$ $\mathrm{kcal} / \mathrm{mol}$. 

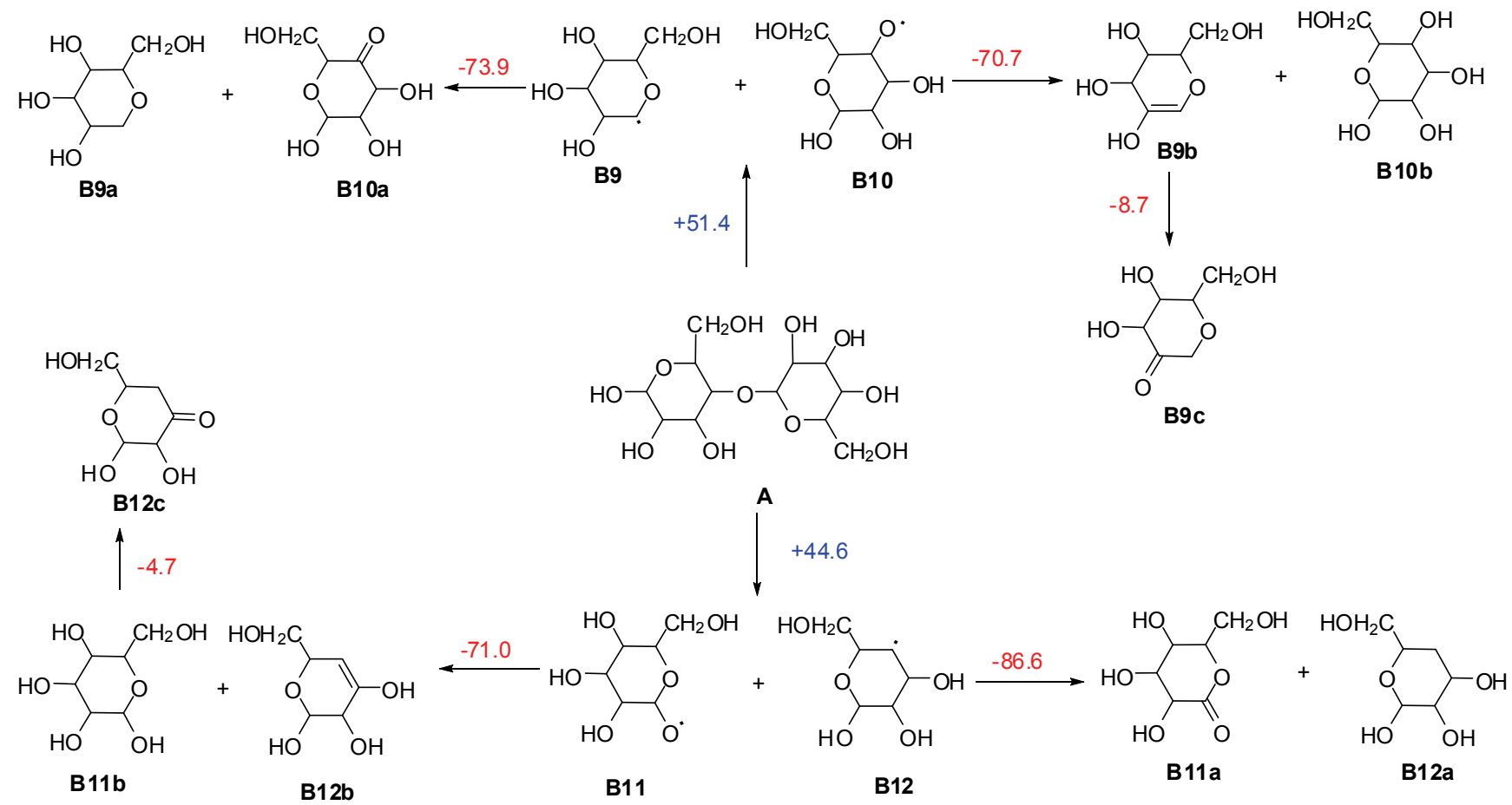

图 3 纤维素二聚体糖苷键断裂过程的自由能 $(\mathrm{kcal} / \mathrm{mol})$ 变化

Figure 3 Free energy ( $\mathrm{kcal} / \mathrm{mol})$ change of glucosidic bond cleavage of cellulose dimer model
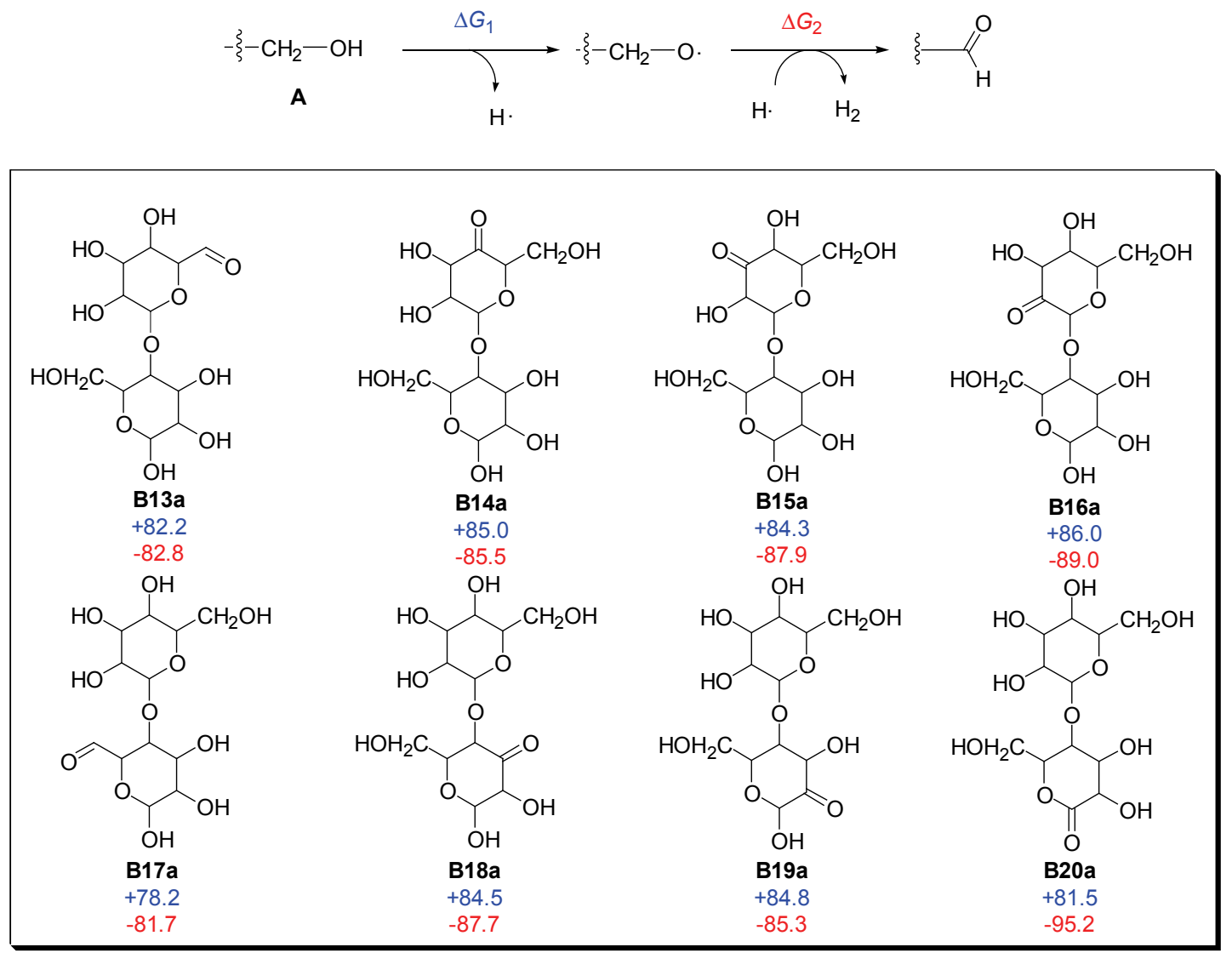

图 4 纤维素二聚 $\mathrm{O}-\mathrm{H}$ 键断裂过程的自由能 $(\mathrm{kcal} / \mathrm{mol})$ 变化

Figure 4 Free energy $(\mathrm{kcal} / \mathrm{mol})$ change of $\mathrm{O}-\mathrm{H}$ bond cleavage of cellulose dimer model 


\section{$3.3 \mathrm{C}-\mathrm{C}$ 键断裂}

然后我们考虑了纤维素二聚体 $\mathbf{A}$ 经历 $\mathrm{C}-\mathrm{C}$ 均裂的 可能性, 如图 5 所示. A 可以通过 $C(5)-C(6)$ 或 $C(5)-$ $\mathrm{C}(6)$ 的均裂分别形成碳自由基 B21 和 B22 以及甲醇自由 基. 两者自由能变接近, 分别为 +46.0 和 +46.1 $\mathrm{kcal} / \mathrm{mol}$. 然后 B21 可以得到由甲醇自由基脱去的氢原 子形成相应的烷烃 B21a，而甲醇形成甲醛，自由能变化 分别为 $-58.8 \mathrm{kcal} / \mathrm{mol}$. B21 也可能脱去 C(4)上的氢原子 给甲醇自由基得到烯烃 B21b 和甲醇, 自由能变化为 $-57.0 \mathrm{kcal} / \mathrm{mol}$. 与形成 B21a 类似, 由 $\mathbf{B 2 2}$ 可以形成 B22a, 自由能变化为 $-59.8 \mathrm{kcal} / \mathrm{mol}$. B22 还可能脱去 $\mathrm{C}(4)$ 上的氢原子形成烯醇, 并异构化为酩 $\mathbf{B 2 2} \mathbf{b}$, 而甲醇<smiles>C[13CH2]c1ccccc1</smiles><smiles>OCC1OC(O)C(O)C(O)C1O</smiles><smiles>CC(C)COC1OC(CO)C(O)C(O)C1OC1=COC(O)C(O)C1O</smiles><smiles>O=C1COCC(O)C1O</smiles>
$\mathrm{OH}$ B21 $\mathrm{CH}_{2} \mathrm{OH}$<smiles>C[13CH3]</smiles><smiles>OCC1OCC(O)C(O)C1O</smiles><smiles>COC1C(O)C(O)C(O)C(O)C1O</smiles>

A<smiles>OCC1C(O)C(O)C(O)C(O)C1OC1OCC(O)C(O)C1O</smiles>

B22

$+$ $\cdot \mathrm{CH}_{2} \mathrm{OH}$

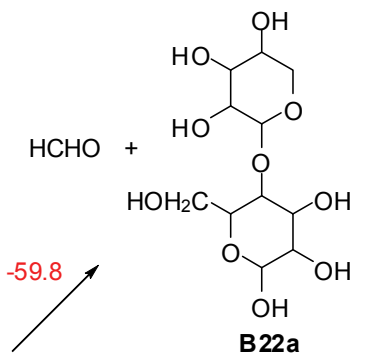

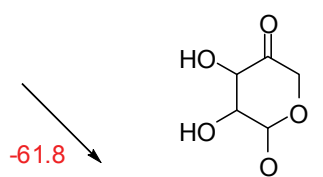
$\mathrm{CH}_{3} \mathrm{OH}+$

B21b

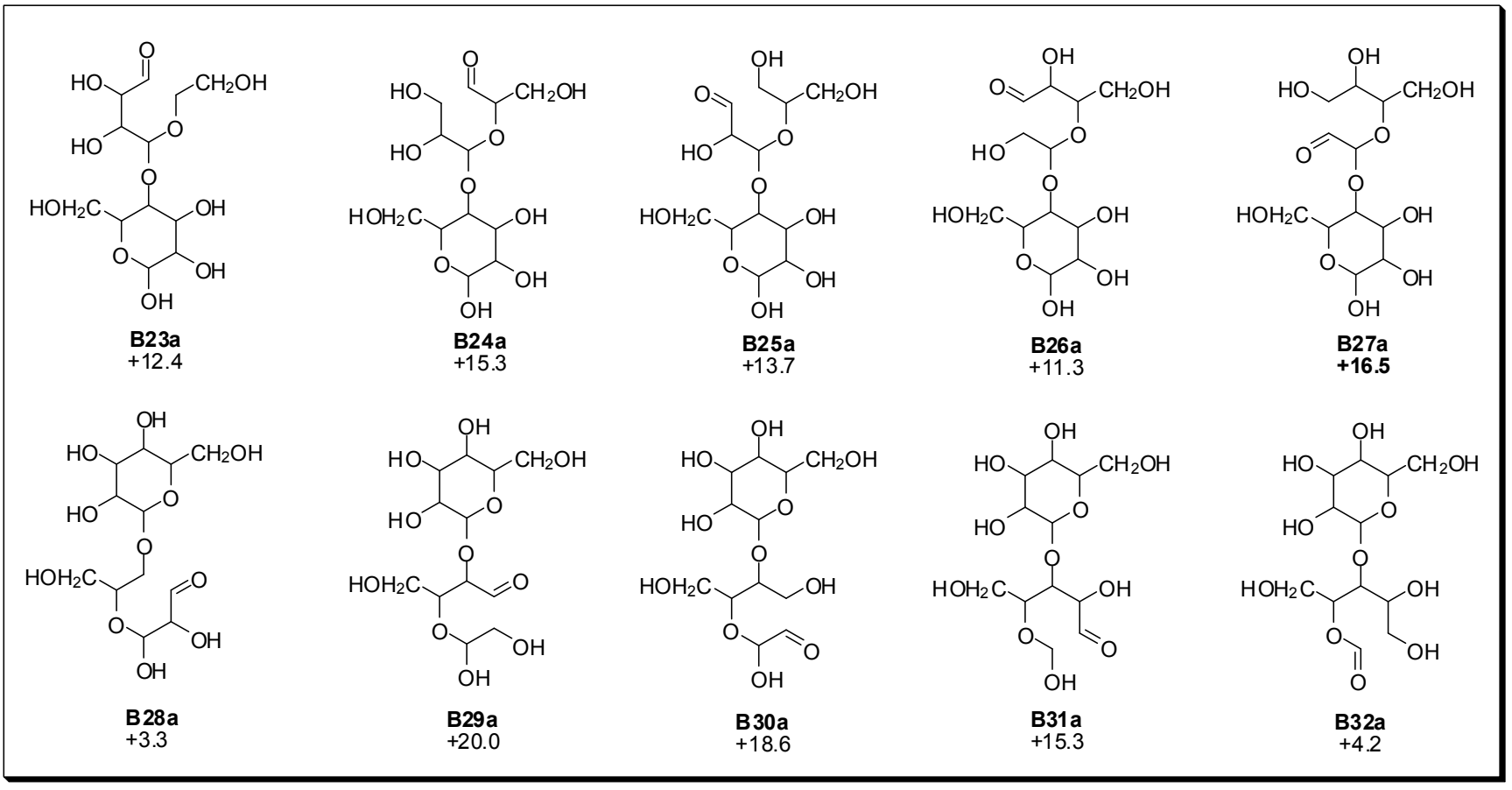

图 5 纤维素二聚 $\mathrm{C}-\mathrm{C}$ 键断裂过程的自由能 $(\mathrm{kcal} / \mathrm{mol})$ 变化

Figure 5 Free energy $(\mathrm{kcal} / \mathrm{mol})$ change of $\mathrm{C}-\mathrm{C}$ bond cleavage of cellulose dimer model 
由能变化分布在 $82 \sim 95 \mathrm{kcal} / \mathrm{mol}$, 而发生侧链 C-C 键 均裂的自由能变化在 $46 \mathrm{kcal} / \mathrm{mol}$ 左右. 因此对于均裂的 过程, 糖苷键的均裂和侧链 $\mathrm{C}-\mathrm{C}$ 键均裂都是更加有利 的过程. 前者将导致纤维素聚合度降低, 而后者可以产 生甲醇和甲醛. 根据 Eyring 公式, 假设反应为一级动力 学反应, 半衰期为 $1 \mathrm{~h}$, 则 $800 \mathrm{~K}$ 条件下对应的反应能垒 为 $62.0 \mathrm{kcal} / \mathrm{mol}$. 故上述两种键均裂也是可能发生的. 对比各种键断裂形成的初级中间产物的稳定性可知, 由 糖苷键断裂形成的中间体产物热力学上最稳定, 其次是 $\mathrm{C}-\mathrm{OH}$ 键均裂过程, 接着是侧链上 $\mathrm{C}-\mathrm{C}$ 键均裂过程. $\mathrm{O}-\mathrm{H}$ 键均裂形成初级中间体在热力学上仅比二聚体略 微稳定, 而环内 $\mathrm{C}-\mathrm{C}$ 键均裂形成初级中间产物则是自 由能升高的过程, 而不能自发进行.

\section{5 产生其它重要小分子的热力学过程}

除了纤维素二聚体不同类型的键均裂热力学变化
外, 我们还对比 Huber 等的实验 ${ }^{[16]}$, 理论动力学计算结 果以及提出的反应路径 ${ }^{[10]}$, 考察了产生其它重要小分 子, 比如 HMF, LGA 等分子的热力学变化情况, 如图 6 所示. 由二聚体 $\mathbf{A}$ 可以经历分子内重排形成四元环结构 B33 和烯醇 B9i, 体系自由能略微升高 $2.7 \mathrm{kcal} / \mathrm{mol}$. B33 再经历一次分子重排可脱出甲酸, 并形成烯醇 B33a. 该 过程导致自由能降低 $35.9 \mathrm{kcal} / \mathrm{mol}$. B9i 异构化为醛 B9k, 则可使体系自由能再降低 $8.4 \mathrm{kcal} / \mathrm{mol}$. 此外由 $\mathbf{A}$ 转化 得到的 B9b 可以经历类似过程得到 HMF 的前体 B9e, 重排过程的自由能变化为 $-0.9 \mathrm{kcal} / \mathrm{mol}$. B9e 再脱去两 分子水可得到 $\mathrm{HMF}$, 该过程自由能变化为 -62.1 $\mathrm{kcal} / \mathrm{mol}$. 此外还可能经由 B9b 形成烯醇类化合物 $\mathbf{B 9 h}$ 和 B9i, 反应自由能变化为 $-19.3 \mathrm{kcal} / \mathrm{mol}$. B9h 和 B9i 可以进一步转化为更稳定的醛或酮 B9k 和 B91, 使体系 自由能再降低 $14.8 \mathrm{kcal} / \mathrm{mol}$. 由 B9b 经过醇氧对烯醇的

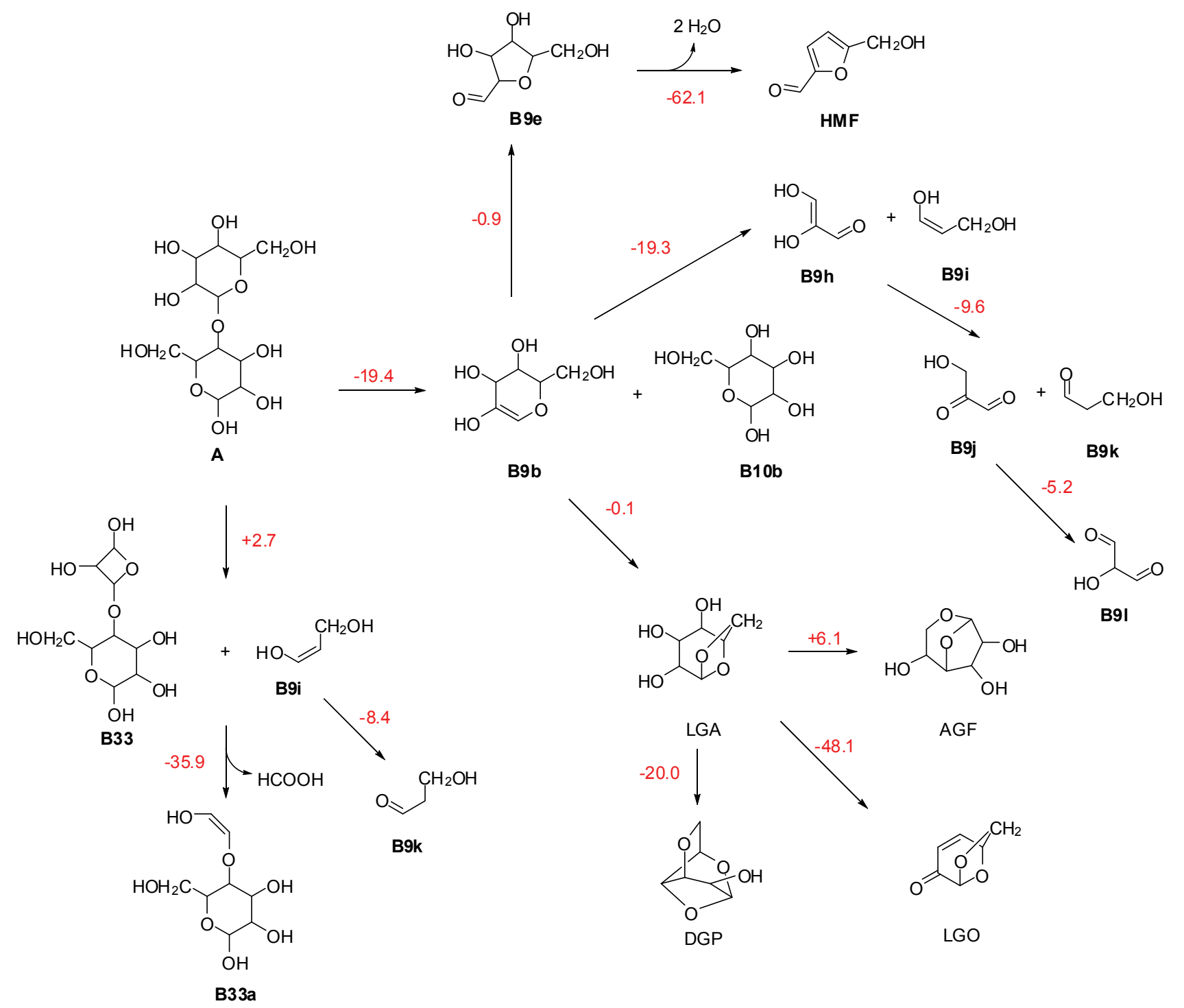

图 6 纤维素二聚体产生重要小分子的自由能 $(\mathrm{kcal} / \mathrm{mol})$ 变化

Figure 6 Free energy (kcal/mol) change of transformations of cellulose dimer into important moleculars 
亲核进攻, 再质子化可形成 LGA, 反应自由能变化近为 $-0.1 \mathrm{kcal} / \mathrm{mol}^{[17]}$. LGA 可以继续转化为其它小分子 $1,6-$ 脱水 $-\beta-D$-呋喃葡萄糖 $\mathrm{AGF}$, 左旋葡萄糖酠 LGO 和 $1,4: 3,6$-二脱水- $\beta$ - $D$-吡喃葡萄糖 DGP. 由 LGA 形成这三 种产物的自由能变化分别为 $+6.1,-48.1$ 和 -20.0 $\mathrm{kcal} / \mathrm{mol}$. 综合各步反应的自由能, 计算结果显示形成 HMF 相较于其它一些小分子, 反应的自由能降低得更 加明显. 其次是 $\mathrm{LGO}$, 总反应放热是 $-67.6 \mathrm{kcal} / \mathrm{mol}$. 接着是形成甲酸和醛类化合物, 自由能放热为 -53.5 和 - $41.6 \mathrm{kcal} / \mathrm{mol}$. 而形成 AGF 相对最不利, 放热仅 $-13.4 \mathrm{kcal} / \mathrm{mol}$. 需要指出的是, 我们不排除以上重要 小分子可以通过其它中间体转化得到的情况. 但在其它 情况下, 如果未转化的部分都转变为了稳定的分子形式 (比如 $\mathrm{H}_{2} \mathrm{O}$ 和 $\mathrm{CO}_{2}$ ) 或同样的化合物, 那么各分子相对的 热力学稳定性也与图 6 结果一致.

对比快速热解实验我们发现, 热力学上的稳定性与 实际产物的比例却没有明显关系(如表 2 所示). 比如在 Huber 的实验中, 并非热力学上最稳定的化合物 LGA 在 所有产物所占比例最高 $(27.9 \%)$, 而热力学上最稳定的 $\mathrm{HMF}$ 的产率仅占 $2.4 \%$. 由 $\mathbf{B 9 b}$ 形成 LGA 是一个熵减少 的过程, 大小为 $-8.4 \mathrm{cal} /(\mathrm{mol} \cdot \mathrm{K})$. 而 $\mathbf{B 9 b}$ 脱去两分子水 形成 $\mathrm{HMF}$ 却是熵明显增加的过程, 大小为 78.9 $\mathrm{cal} /(\mathrm{mol} \cdot \mathrm{K})$. 高温度条件下形成 LGA 在热力学上不利, 而形成 HMF 有利这一结果是合理的. 具体来说, 在 800 $\mathrm{K}$ 的条件下, B9b 形成 $\mathrm{HMF}$ 的自由能变化为 -63.0 $\mathrm{kcal} / \mathrm{mol}$, 形成 LGA 的自由能变化仅 $-0.1 \mathrm{kcal} / \mathrm{mol}$, 两 者相差 $62.9 \mathrm{kcal} / \mathrm{mol}$. 而在标准条件 $298 \mathrm{~K}$ 的情况下, B9b 形成 HMF 的自由能变化为 $-22.8 \mathrm{kcal} / \mathrm{mol}$, 形成 LGA 的自由能变化为 $-3.9 \mathrm{kcal} / \mathrm{mol}$, 仅相差 18.9 $\mathrm{kcal} / \mathrm{mol}$. 尽管常温和高温条件下都显示形成 HMF 在热 力学上占优势, 然而实验中 HMF 仅占很少的一部分. 造成这现象的原因可能是如 Huber 等的动力学研究结果 所揭示的, 形成 LGA 前体比形成 HMF 前体在动力学上 更占优势. 所以尽管 HMF 要明显更加稳定, 但在产物 中所占比例更少. 有趣的是, 我们发现调节温度甚至可 以使反应由可自由能降低过程变为自由能升高过程. 比 如 $800 \mathrm{~K}$ 条件下, 由 $\mathbf{A}$ 形成 $\mathrm{AGF}$ 是可自发进行的, 但在 $298 \mathrm{~K}$ 的条件下却不可以. 由此可见熵效应和温度对不 同组分热力学的稳定性差异造成的巨大影响 ${ }^{[18]}$.

此外 LGA 也比其后续脱水产物 LGO 和 DGP 所占 比例大. 我们认为可能是热解反应不充分导致的. Huber 的实验中纤维素的热解从 $323 \mathrm{~K}$ 开始以每分钟升高 150 $\mathrm{K}$ 的速率加热至 $1073 \mathrm{~K}$, 然后在 $1073 \mathrm{~K}$ 的条件下再维 持 $6 \mathrm{~min}$, 总反应时间约为 $11 \mathrm{~min}$. 在这期间, 有相当一 部分时间是在发生纤维素由高聚物降解为低聚物的过 程, 而后续的转化过程可能仅占其中的一部分时间. 所 以最终结果是中间产物 LGA 更多, 而后续脱水产物 LGO 或 DGP 较少.
表 2 反应自由能变化与快速热解实验对比

Table 2 Comparison of free energy change and fast pyrolysis experiment

\begin{tabular}{lccc}
\hline Compd. & 比例 $/ \%$ & $\Delta G_{800 \mathrm{~K}}$ & $\Delta G_{298 \mathrm{~K}}$ \\
\hline LGA & 27.9 & -19.5 & +1.9 \\
HMF & 2.4 & -82.4 & -17.0 \\
AGF & 3.3 & -13.4 & +8.6 \\
LGO & 5.5 & -67.6 & -7.5 \\
DGP & 3.3 & -39.5 & -1.8 \\
Aldehyde (B9k+B34a) & - & -53.5 & -3.8 \\
HCOOH & - & -41.6 & +7.9 \\
\hline
\end{tabular}

通过上述比较说明热力学计算结果在复杂反应过 程或非平衡状态下并不适合于分析反应产物成分. 有趣 的是, 我们发现温度的变化对反应有重要的影响. 对于 分子数增多的过程通常也是熵增的过程, 升高温度可促 进该过程. 反之对分子重排过程，商的变化却不明显， 温度对该类过程的影响则较小 ${ }^{[19]}$. 这样, 通过对温度的 控制有可能实现在动力学和热力学上对熵变化不同的 反应过程进行调控, 从而选择性地得到某类产物.

\section{4 结论}

了解纤维素热解机理对发展有效的生物质转化技 术有重要意义. 我们利用密度泛函理论方法, 以纤维素 二聚体为模型研究了其热解过程的热力学性质. 计算结 果显示纤维素二聚体发生糖苷键和侧链 $\mathrm{C}-\mathrm{C}$ 键的均裂 比其它 $\mathrm{C}-\mathrm{O}$ 键和 $\mathrm{O}-\mathrm{H}$ 键的均裂更加容易. 并且通过 糖苷键均裂过程形成的初级中间产物比其它键均裂过 程形成的产物更加稳定. 通过对比重要小分子的热力学 稳定性和快速热解实验结果, 发现产物的热力学稳定性 并不能正确反映其在快速热解实验中的实际比例. 故不 推荐单独使用热力学性质去分析快速非平衡或复杂反 应过程中的产物成分. 通过考察不同温度下热力学变化 情况，发现温度对熵变不同的过程的重要影响，为通过 温度来调控熵变化不同的反应，进而选择性得到某类产 物提供了一条思路.

致谢 本文中所有计算均在中国科学技术大学超级计 算中心及上海超级计算中心完成并受中国教育科研网 格 ChinaGrid 资助.

\section{References}

[1] Gellerstedt, G.; Henriksson, G. In Monomers, Polymers and Composites from Renewable Resources, Eds.: Belgacem, M.; Gandini, A., Elsevier, Amsterdam, 2008, pp. 201 224.

[2] (a) Bridgwater, A. V.; Peacocke, G. V. C. Renew. Sust. Energy Rev. 2000, 4, 1. (b) Corma, A.; Iborra, S.; Velty, A. Chem. Rev. 2007, 107, 2411. (c) Climent, M. J.; Corma, A.; Iborra, S. Green Chem. 2011, 13, 520. (d) Carlson, T. R.; Vispute, T. P.; Huber, G. W. ChemSusChem 2008, 1, 397.

[3] Agarwal, V.; Huber, G. W.; Conner, W. C.; Auerbach, S. M. J. Chem. Phys. 2011, 135, 134506. 
[4] (a) Wang, J.; Zhang, M. X.; Chen, M. Q.; Min, F. F.; Wang, X. Y.; Shao, Q.; Zhang, X. C.; Zhang, S. P.; Ren, Z. W.; Yan, Y. J. J. China Coal Soc. 2007, 32, 194. (王君, 张明旭, 陈明强, 闵凡飞, 王新运, 邵群, 张学才, 张素平, 任铮伟, 颜涌捷, 煤炭学报, 2007, 32, 194.) (b) Yang, H. M.; Han, C. L.; Wu, Y. P.; Bi, Y.; Yin, G. M. J. Sci. Teachers' College Univ. 2008, 3, 57. (杨海明, 韩成 利, 吴也平, 毕野, 殷广明, 高师理科学刊, 2008, 3, 57.) (c) Fachuang, L.; John, R. J. Agric. Food Chem. 1997, 45, 4655. (d) Fachuang, L.; John, R. J. Agric. Food Chem. 1998, 46, 547.

[5] Mamleev, V.; Bourbigot, S.; Le Bras, M.; Yvon, J. J. Anal. Appl. Pyrolysis 2009, 84, 1.

[6] Lanza, R.; Dalle Nogare, D.; Canu, P. Ind. Eng. Chem. Res. 2009, $48,1391$.

[7] (a) Lédé, J.; Blanchard, F.; Boutin, O. Fuel 2002, 81, 1269. (b) Boutin, O.; Ferrer, M.; Lédé, J. Chem. Eng. Sci. 2002, 57, 15.

[8] (a) Qiao, Z. P.; Wang, H.; Zhao, W. L.; Ran, X. Q. J. Fuel Chem. Technol. 2002, 1, 49. (乔占平, 王惠, 赵文立, 由新权, 燃料化学 学报, 2002, 1, 49.) (b) Wang, H.; Zhao, Y.; Wang, C.; Fu, Y.; Guo, Q. Acta Chim. Sinica 2009, 67, 893. (王华静, 赵岩, 王晨, 傅尧, 郭庆祥，化学学报, 2009, 67, 893.) (c) Wang, H.; Yang, H. F.; Zhai, G. H.; Wen, Z. Y.; Ran, X. Q.; Shi, Q. Z. Acta Chim. Sinica 2001, 59, 17. (王惠, 杨海峰, 翟高红, 文振翼, 再新权, 史启祯, 化学 学报, 2001, 59, 17.) (d) Wang, H. X.; Peng, Q. J.; Luo, D. M.; Zen, Y. Acta Chim. Sinica 2003, 61, 1577. (主辉宪, 彭清静, 罗明道, 曾跃, 化学学报, 2003, 61, 1577.)

[9] Zhang, X.; Yang, W.; Blasiak, W. J. Anal. Appl. Pyrolysis 2012, 96, 110.

[10] Agarwal, V.; Dauenhauer, P. J.; Huber, G. W.; Auerbach, S. M. J. Am. Chem. Soc. 2012, 134, 14958.

[11] (a) He, J.; Zhao, C.; Lercher, J. A. J. Am. Chem. Soc. 2012, 134, 20768. (b) Jiao, Y.; Evans, M. E.; Morris, J.; Brennessel, W. W.; Jones, W. D. J. Am. Chem. Soc. 2013, 135, 6994. (c) Wang, M.; Fan, T.; Lin, Z. Organometallics 2012, 31, 560. (d) Wang, H.; Luo, R. Y.; Yang, Y. Q.; Ran, X. Q.; Wen, Z. Y. Chin. J. Mater. Res. 2001, 15, 159. (王惠, 罗瑞盈, 杨延清, 申新权, 文振翼, 材料研究学 报, 2001, 15, 159.) (e) Zhai, G. H.; Zhu, K. K.; Wang, H.; Ran, X. Q.; Shi, Q. Z.; Wen, Z. Y.; Luo, R. Y.; Yang, Y. Q. Mater. Sci. Eng. 2000, 18, 10. (翟高红, 朱卡克, 王惠, 冉新权, 史启桢, 文振翼, 罗瑞盈, 杨延清, 材料科学与工程, 2000, 18, 10.) (f) Wang, H.; Zhai, G. H.; Ran, X. Q.; Shi, Q. Z.; Wen, Z. Y.; Luo, R. Y.; Yang, Y. Q. Chin. J. Inorg. Chem. 2000, 16, 879. (王惠, 翟高红, 手新权, 史启桢，文振翼，罗瑞盈，杨延清，无机化学学报, 2000, 16, 879.)

[12] Frisch, M. J.; Trucks, G. W.; Schlegel, H. B.; Scuseria, G. E.; Robb, M. A.; Cheeseman, J. R.; Scalmani, G.; Barone, V.; Mennucci, B.;
Petersson, G. A.; Nakatsuji, H.; Caricato, M.; Li, X.; Hratchian, H. P.; Izmaylov, A. F.; Bloino, J.; Zheng, G.; Sonnenberg, J. L.; Hada M.; Ehara, M.; Toyota, K.; Fukuda, R.; Hasegawa, J.; Ishida, M.; Nakajima, T.; Honda, Y.; Kitao, O.; Nakai, H.; Vreven, T.; Montgomery, J. A. Jr.; Peralta, J. E.; Ogliaro, F.; Bearpark, M.; Heyd, J. J.; Brothers, E.; Kudin, K. N.; Staroverov, V. N.; Keith, T.; Kobayashi, R.; Normand, J.; Raghavachari, K.; Rendell, A.; Burant, J. C.; Iyengar, S. S.; Tomasi, J.; Cossi, M.; Rega, N.; Millam, J. M.; Klene, M.; Knox, J. E.; Cross, J. B.; Bakken, V.; Adamo, C.; Jaramillo, J.; Gomperts, R.; Stratmann, R. E.; Yazyev, O.; Austin, A. J.; Cammi, R.; Pomelli, C.; Ochterski, J. W.; Martin, R. L.; Morokuma, K.; Zakrzewski, V. G.; Voth, G. A.; Salvador, P.; Dannenberg, J. J.; Dapprich, S.; Daniels, A. D.; Farkas, O.; Foresman, J. B.; Ortiz, J. V.; Cioslowski, J.; Fox, D. J. Gaussian 09, Revision B. 01. Gasussian Inc., Wallingford CT, 2010.

[13] (a) Zhao, Y.; Truhlar, D. G. Theor. Chem. Acc. 2008, 120, 215. (b) Zhao, Y.; Truhlar, D. G. Acc. Chem. Res. 2008, 41, 157.

[14] (a) Feng, Y.; Liu, L.; Wang, J. T.; Huang, H.; Guo, Q. X. J. Chem. Inf. Comput. Sci. 2003, 43, 2005. (b) Feng, Y.; Liu, L.; Wang, J. T.; Zhao, S. W.; Guo, Q. X. J. Org. Chem. 2004, 69, 3129. (c) Song, K. S.; Liu, L.; Guo, Q. X. J. Org. Chem. 2003, 68, 262. (d) Cheng, Y. H.; Zhao, X.; Song, K. S.; Liu, L.; Guo, Q. X. J. Org. Chem. 2002, 67,6638 .

[15] Due to structural limitation, the $\mathrm{C}-\mathrm{C}$ bonds on the carbocyclic ring will spontaneous reform after we break them during geometry optimization. Therefore the hemolytic bond dissociation energy for those bonds cannot be calculated.

[16] Lin, Y.-C.; Cho, J.; Tompsett, G. A.; Westmoreland, P. R.; Huber, G. W. J. Phys. Chem. C 2009, 113, 20097.

[17] The presented results did not consider the reactions of B10b whereas it will further transform into more stable species. Thus the energy change from B9b to B9e or LGA is more exothermic in real pyrolysis reaction.

[18] (a) Liu, L.; Guo, Q. X. Chem. Rev. 2001, 101, 673. (b) Liu, L.; Yang, C.; Guo, Q. X. Biophys. Chem. 2000, 84, 239.

[19] It is noted that the contribution of entropy is usually over estimated, and the following literatures can be referred to solve this problem: (a) Liu, Q.; Lan, Y.; Liu, J.; Li, G.; Wu, Y.-D.; Lei, A. J. Am. Chem. Soc. 2009, 131, 10201. (b) Schoenebeck, F.; Houk, K. N. J. Am. Chem. Soc. 2010, 132, 2496. (c) Ariafard, A.; Brookes, N. J.; Stranger, R.; Yates, B. F. Organometallics 2011, 30, 1340. (d) Liu, B.; Gao, M.; Dang, L.; Zhao, H.; Marder, T. B.; Lin, Z. Organometallics 2012, 31, 3410 . 